PROCEEDINGS OF THE

AMERICAN MATHEMATICAL SOCIETY

Volume 129, Number 12, Pages 3701-3709

S 0002-9939(01)06008-7

Article electronically published on April 25, 2001

\title{
DECOMPOSITION OF SPACES WITH GEODESICS CONTAINED IN COMPACT FLATS
}

\author{
BERNARDO MOLINA AND CARLOS OLMOS \\ (Communicated by Christopher Croke)
}

\begin{abstract}
We prove a decomposition result for analytic spaces all of whose geodesics are contained in compact flats. Namely, we prove that a Riemannian manifold is such a space if and only if it admits a (finite) cover which splits as the product of a flat torus with simply connected factors which are either symmetric (of the compact type) or spaces of closed geodesics.
\end{abstract}

\section{INTRODUCTION}

In a previous article $\mathrm{MO}$ the authors obtained a higher rank rigidity characterization of compact locally symmetric spaces which might be interpreted as a dual result to the remarkable theorem of Ballmann [B] and Burns-Spatzier [BS] (see also $[\mathrm{EH}]$ ). Namely, they obtained the following result.

Theorem ([M]). Let $M$ be a complete Riemannian manifold that is locally irreducible at every point of some open set. Assume that there exists an open and dense subset $\Omega$ of TM such that for any $w \in \Omega$ the geodesic $\gamma_{w}$ is contained in a compact (immersed) flat of dimension at least 2 . Then, $M$ is a locally symmetric space of the compact type.

Observe that, on one hand, no curvature assumptions are made. On the other hand, the flats are assumed to be compact. Thus, the duality seems to have nothing to do with reversing the sign of curvature, as an abundant number of counterexamples due to Heintze and Spatzier-Strake [SS] show. This duality is concerned with the global behavior of geodesics which is, in some sense, the opposite to that for nonpositive curvature and finite volume. Just notice that the geodesic flow for this class of spaces, when the rank is 1 , is expected to be ergodic. But this flow, for spaces all of whose geodesics are closed, is as far as it can be from having this property. In contrast to the case of nonpositive curvature, a global decomposition result for spaces with geodesics contained in compact flats is far from being trivial. This is so because, in this context, one cannot integrate the distribution (in the tangent space) associated to the notion of rank in terms of Jacobi fields (and producing in this way flats whose dimensions are equal to the rank). For analytic

Received by the editors December 16, 1999 and, in revised form, April 17, 2000.

1991 Mathematics Subject Classification. Primary 53C35; Secondary 53C20.

Key words and phrases. Compact flats, rank rigidity, holonomy.

Supported by Universidad Nacional de Córdoba, CONICET and DAAD, partially supported by CONICOR, Secyt-UNC and CIEM. 
manifolds with compact universal cover, this decomposition can be obtained in a standard way from the above theorem (see [MO]). The aim of this article is to prove the following general decomposition result for the analytic case (in fact, the same applies to the general case provided the space is not too pathological).

Theorem A. Let $M$ be an analytic (complete) Riemannian manifold. Then the following statements are equivalent:

(i) Every geodesic in $M$ is contained in a compact flat.

(ii) $M$ admits a (finite) cover of the form $T^{k} \times C_{1} \times \cdots \times C_{r} \times S_{1} \times \cdots \times S_{l}$, where $T^{k}$ is a flat torus, $C_{i}$ is a (simply connected) space all of whose geodesics are closed, and $S_{j}$ is a (simply connected) irreducible symmetric space of the compact type and rank at least $2(i=1, \ldots, r, j=1, \ldots, l)$.

From the analyticity and the proof it is easy to obtain that we can replace, in the above result, the words "every geodesic" by "every geodesic with initial condition in an open and nonempty subset of $T M$ ". We conjecture that the above theorem is true without assuming analyticity (this is a stronger version of the conjecture posed in $[\mathrm{MO}$, but it turns out to be equivalent as was remarked in section 4). We now sketch the proof of Theorem A. The first step is to show that the universal cover is compact up to a Euclidean factor, and each of the compact factors is a higher rank symmetric space or a space all of whose geodesics are closed. We do this by proving that factors with transitive holonomy must have all of their geodesics closed. After this we pass to an intermediate but convenient cover in which the covering group is abelian and completely determined by its projection to the Euclidean factor. If the projection of this group to the other factor were infinite, there would be a nontrivial Killing field corresponding to a nonzero element in the Lie algebra of the closure of this group. We show then that the projection of this Killing field to any non-Euclidean factor, at a given point, would be contained in any flat through this point. This is impossible, unless $X=0$, because the factors are either irreducible symmetric or of closed geodesics.

The rank, in terms of parallel Jacobi fields, must be 1 for a space all of whose geodesics are closed (see Remark 4.2). On the other hand, any geodesic in a compact symmetric space of rank $k$ is contained in a compact $k$-flat. Then, Theorem A can be reformulated as follows:

Theorem B. Let $M$ be an analytic Riemannian manifold. Then, the following statements are equivalent:

i) Every geodesic of $M$ is contained in a compact flat.

ii) Every geodesic of $M$ is contained in a compact $k$-flat, where $k=\operatorname{rank}(M)$.

iii) $M$ is covered by a space which is the product of a compact globally symmetric space with spaces all of whose geodesics are closed.

The above theorem suggests to find a general global condition on $M$ which implies that the horizontal distribution in $T M$ associated to the rank be integrable with compact leaves (observe that in [SS] there are examples of irreducible higher rank nonsymmetric compact spaces of nonnegative curvature and such that the distribution associated to the rank is integrable).

We hope that some ideas in this article could be useful for proving Theorem A without analyticity assumptions. But the main difficulty is that the space could be, a priori, so pathological that it is locally reducible at any point and globally 
irreducible. So, the local version of the holonomy theorem of Berger $[\mathrm{Be}, \mathrm{S}]$ cannot be applied, as in $\mathrm{MO}$.

\section{PReliminaries and Basic FaCts}

In this section we recall some of the results and notation in $\mathrm{MO}$. We also make a remark which not only simplifies the arguments there, but is also used in this article.

Let $\left(M^{n},<,>\right)$ be a complete connected Riemannian manifold. We say that $M$ is locally reducible at $p \in M$ if $p$ has an open neighborhood in $M$ which is a Riemannian product. This is equivalent to the fact that the local holonomy group at $p$ does not act irreducibly on $T_{p} M$. If $v \in T_{p} M, \gamma_{v}$ denotes the (complete) geodesic defined by $\gamma_{v}^{\prime}(0)=v$. A flat containing $\gamma_{v}$ is an isometric totally geodesic immersion $i: F \rightarrow M$, where $F$ is a complete connected flat Riemannian manifold, such that $\gamma_{v}(\mathbb{R})$ is contained in $i(F)$. We say that the flat is compact if $F$ is compact and that the flat is toral if $F$ is homeomorphic to a torus. Since any compact flat manifold is covered by a torus, a geodesic is contained in a compact flat if and only if it is contained in a toral one. A $k$-flat means a flat of dimension $k(k \in \mathbb{N})$. Sometimes a flat $i: F \rightarrow M$ will be denoted by the pair $(F, i)$ or simply by $i$ or by $F$ when the immersion is obvious from the context. We say that a flat $i: F \rightarrow M$ has no self intersection at $q \in i(F)$ if $d i\left(T_{x} F\right)$ does not depend on $x \in i^{-1}(q)$. In this case the uniquely defined subspace $d i\left(T_{x} F\right)$ of $T_{q} M$ will be called the tangent space to the flat at $q$.

Let $v(t), t \in[0,1]$, be a smooth curve in $T M$ and let $c(t)$ be its projection to $M$. Let, for $t \in[0,1],\left(F(t), i_{t}\right)$ be a toral flat containing the geodesic $\gamma_{v(t)}$. We assume that the flat space $F(t)$ has a distinguished point $u(t)$ such that $i_{t}(u(t))=c(t)$. We say that the family of flats $\left(F(t), i_{t}\right)$ vary smoothly if there exist affine maps $h_{t}: F(0) \rightarrow F(t)$ with $h_{t}(u(0))=u(t), h_{0}=I d$, and such that $g:[0,1] \times F(0) \rightarrow M$ is smooth, where $g_{t}=i_{t} \circ h_{t}$.

Remark 1.1. We are under the above hypothesis. Assume furthermore that $v(t)$ is horizontal, i.e. it is parallel along its projection $c(t)$. Then the affine map $h_{t}$ : $F(0) \rightarrow F(t)$ is an isometry. In fact, if we choose a closed geodesic $\gamma$ in $F(0)$, then $g_{t} \circ \gamma$ is a smooth variation of closed geodesics in $M$. Since any geodesic is a minimal submanifold, all of these geodesics must have the same length. Then, $\left\|d g_{t}\left(\gamma^{\prime}(0)\right)\right\|$ is constant and therefore $\left\|d h_{t}\left(\gamma^{\prime}(0)\right)\right\|$ is constant. Since closed geodesics in a torus are dense, we have that $h_{t}$ is an isometry. This simplifies the arguments in [MO] section 5]. Namely, the equality (I) there and Cauchy-Schwarz inequality implies that

$$
d g_{1}\left(\hat{v}_{0}\right)=v(1)
$$

where $\hat{v}_{0} \in T_{u(0)} F(0)$ and $d i_{0}\left(\hat{v}_{0}\right)=v(0)$. In fact, it is proved in section 4 of $\mathrm{MO}$ ] that the covariant derivative $\frac{D}{d t} d g_{t}\left(\hat{v}_{0}\right)$ is always perpendicular to $d i_{t}\left(T_{u(t)} F(t)\right)$

and therefore to $v(t)$. Then, $\frac{d}{d t}\left\langle d g_{t}\left(\hat{v}_{0}\right), v(t)\right\rangle=\left\langle\frac{D}{d t} d g_{t}\left(\hat{v}_{0}\right), v(t)\right\rangle=0$. Equality (*) follows now from Cauchy-Schwarz inequality, since $d g_{t}\left(\hat{v}_{0}\right)$ and $v(t)$ have both constant length and $d g_{0}\left(\hat{v}_{0}\right)=v(0)$.

The remark above, the Regularity Lemma (where the dimension of the flats is irrelevant) and Lemma 2.2 in $\mathrm{MO}$ can be summarized as follows. 
Lemma 1.2. Let $\tilde{\mathcal{O}}$ be an open subset of $T M$ with the property that every geodesic with initial condition in $\tilde{\mathcal{O}}$ is contained in a compact flat. Then, there exists an open nonempty subset $\mathcal{O}$ of $\tilde{\mathcal{O}}$ and a choice of toral $k$-flats $i_{v}: F_{v} \rightarrow M$ containing the geodesic $\gamma_{v}, v \in \mathcal{O}$ (with distinguished points $u(v)$, with $i_{v}(u(v))=\gamma_{v}(0)$ ), and having the following properties:

i) The flat $\left(F_{v}, i_{v}\right)$ has no self intersection at $\pi(v)$, for any $v \in \mathcal{O}$.

ii) $\mathcal{F}$ defines a $C^{\infty}$ distribution on $\mathcal{O}$, where $\mathcal{F}_{v}$ is the unique subspace of the horizontal subspace $\mathcal{H}_{v}$ such that $d \pi\left(\mathcal{F}_{v}\right)$ coincides with the tangent space to the flat at $\pi(v)$.

iii) If $v(t)$ is a curve in $\mathcal{O}$, then there is a smooth choice of isometric toral flats $\left(F(t), i_{t}\right)$, containing the geodesic $\gamma_{v(t)}$ and that $i_{t}(F(t))=i_{v(t)}\left(F_{v(t)}\right)$.

It is easy to see that the lemma in Section 5 of $\mathrm{MO}$ is still valid for any symmetric factor of the universal cover. In fact, the variation of geodesics, used in the proof of that result, has only to be carried out in such a factor. Thus, one has the following generalization.

Lemma 1.3. Let $M^{n}$ be a complete Riemannian manifold such that every geodesic in $M$, with initial condition in an open and nonempty subset of $T M$, is contained in a compact flat. Then, any non-Euclidean symmetric factor of the universal cover of $M$ is of the compact type.

\section{Decomposition of the universal COVERing}

The aim of this section is to prove the following proposition which generalizes Theorem B in [MO]. This will be one of the main two steps for proving the theorem in the introduction.

Proposition 2.1. Let $M^{n}$ be an analytic complete Riemannian manifold such that every geodesic, with initial condition in a (nonempty) open subset of TM, is contained in a compact immersed flat. Then the universal covering $\tilde{M}$ of $M$ splits as $\tilde{M}=\mathbb{R}^{k} \times C_{1} \times \cdots \times C_{r} \times S_{1} \times \cdots \times S_{l}$, where $C_{i}$ is a (simply connected) space all of whose geodesics are closed and $S_{j}$ is a (simply connected) irreducible symmetric space of the compact type and rank at least $2(i=1, \ldots, r, j=1, \ldots, l)$.

Proof. Let $X$ be a non-Euclidean factor of $\tilde{M}$. If the holonomy group of $X$ (at some point) does not act transitively on the unit sphere of the tangent space, then, by $[B]$, $[\mathrm{S}, X$ is a symmetric space of higher rank which must be compact due to Lemma 1.3. So, let us then assume that this action is transitive and write $\tilde{M}=M_{0} \times X$. Let $\mathcal{O} \subset T M$ be the subset given by Lemma 1.2, let $v \in \mathcal{O}$ with base point $p$, and let $\tilde{v}=\left(\tilde{v}_{0}, \tilde{v}_{1}\right) \in T M_{0} \times T X=T \tilde{M}$ be such that $d \pi(\tilde{v})=v$, where $\pi: \tilde{M} \rightarrow M$ is the covering map. Let us denote by $\tilde{p}=\left(\tilde{p}_{0}, \tilde{p}_{1}\right) \in M_{0} \times X=\tilde{M}$ the base point of $\tilde{v}$. Since $\mathcal{O}$ is open and closed geodesics in a torus are dense, we may assume, without loss of generality, that $\tilde{v}_{1} \neq 0$ and that the geodesic $\gamma_{v}$ in $M$ is closed. Let $\varepsilon>0$ be such that the geodesic $\varepsilon$-ball in $T M$ (with respect to the Sasaki metric), centered at $v$, is contained in $\mathcal{O}$. (Observe that the parallel transport of $v$ along a curve of length less than $\varepsilon$ may be also thought as a (horizontal) curve in $T M$ of length less than $\varepsilon$.) Let $\Phi$ be the holonomy group (which coincides, by the analyticity, with the local one) of $X$ at $\tilde{p}_{1}$ and let $U$ be the subset of $\Phi$ which consists of all the parallel transport along (piecewise differentiable) loops through $\tilde{p}_{1}$ of length less than $\varepsilon$ (i.e. $\varepsilon$-loops). Then by EO, Appendix] $U$ contains an 
open neighborhood $W$ of the identity of $\Phi$ (though in this reference piecewise $C^{1}$ loops are used, the same proof applies for piecewise differentiable ones). We may assume, by making $W$ smaller if necessary, that $d \pi\left(v_{0}, W \cdot \tilde{v}_{1}\right) \subset \mathcal{O}$. Observe that $W . \tilde{v}_{1}$ is an open neighborhood of $\tilde{v}_{1}$ in the sphere (of radius $\left|\tilde{v}_{1}\right|$ ) of $T_{\tilde{p}_{1}} X$. Now let $w \in W \cdot \tilde{v}_{1}$ and let $\tilde{\beta}_{w}$ be an $\varepsilon$-loop through $\tilde{p}_{1}$ such that its parallel transport maps $\tilde{v}_{1}$ into $w$. Let $\beta_{w}(t)=\pi\left(\tilde{p}_{0}, \tilde{\beta}_{w}(t)\right)$ and $\bar{v}(t)=d \pi\left(\tilde{v}_{0}, w(t)\right)$, where $w(t)$ is the parallel transport of $\tilde{v}_{1}$ along $\tilde{\beta}_{w}$. Observe that $\bar{v}(t)$ is parallel along $\beta_{w}(t)$ with $\bar{v}(0)=v$. We can produce, by Lemma 1.2 (iii) (see also [M]), a smooth variation $g_{t}: F(0) \rightarrow F(t)$ of compact immersed flats $F(t)$ containing the geodesic $\gamma_{\bar{v}(t)}$. We have, by Remark 1.1, that $\bar{v}(1)=d g_{1}(v)$ and therefore the geodesic $\gamma_{\bar{v}(1)}$ is closed with the same period (not perhaps the least one), let us say $l$, of $\gamma_{v}$. Then, $\pi\left(\gamma_{\left(\tilde{v}_{0}, w\right)}(l)\right)=\pi\left(\gamma_{\left(\tilde{v}_{0}, \tilde{v}_{1}\right)}(l)\right)$, or equivalently,

$$
\pi \circ \exp _{\tilde{p}}\left(l \cdot\left(\tilde{v}_{0}, \tilde{v}_{1}\right)\right)=\pi \circ \exp _{\tilde{p}}\left(l \cdot\left(\tilde{v}_{0}, w\right)\right)
$$

for all $w \in W . \tilde{v}_{1}$. Since $W . \tilde{v}_{1}$ is an open subset of the sphere $S_{\left\|\tilde{v}_{1}\right\|}$ of radius $\left\|\tilde{v}_{1}\right\|$ in $T_{\tilde{v}_{1}} X$, by the analyticity, if $\left(m_{0}, m(w)\right):=\left(\exp _{\tilde{p}_{0}}\left(l . \tilde{v}_{0}\right), \exp _{\tilde{p}_{1}}(l . w)\right)$, we have that $\pi\left(m_{0}, m(w)\right)$ does not depend on $w$ in $S_{\left\|\tilde{v}_{1}\right\|}$. Since $\pi^{-1}\left(\pi\left(m_{0}, m(w)\right)\right)$ is a discrete subset of $\tilde{M}$, we obtain that the continuous map $w \mapsto m(w)$ must be constant, let us say $\tilde{q}$, on $S_{\| \tilde{v}_{1}||}$. So, any unit speed geodesic in $X$ starting at $\tilde{p}$ must pass through $\tilde{q}$ at the time $l\left\|\tilde{v}_{1}\right\|$. This implies that there cannot be a ray starting at $\tilde{p}$. Hence $X$ is compact. We now want to see that the geodesics of $X$ are closed. Let $\tilde{\gamma}$ be a geodesic in $X$ and let $\operatorname{cl}(\tilde{\gamma}(\mathbb{R}))$ be the closure in $X$ of (the image of) the geodesic $\tilde{\gamma}$. Since $X$ is compact, we get that $\pi(\operatorname{cl}(\tilde{\gamma}(\mathbb{R})))$ is closed in $M$. Since $\pi(\operatorname{cl}(\tilde{\gamma}(\mathbb{R})))$ must be contained in $\operatorname{cl}(\gamma(\mathbb{R}))$, we obtain that both subsets of $M$ coincide $(\gamma=\pi \circ \tilde{\gamma})$. We have, from the assumptions, that $\operatorname{cl}(\gamma(\mathbb{R}))$ is a compact flat in $M$. It is now not hard to see, from the compactness of $X$, that $c l(\tilde{\gamma}(\mathbb{R}))$ is a compact (immersed) flat in $X$ containing the geodesic $\tilde{\gamma}$. So, any geodesic in $X$ is contained in a compact flat. Then, by the analyticity of $X$ and by Remark 6.7 of [MO], $X$ must be a space all of whose geodesics are closed due to the transitivity of the holonomy group.

\section{Nonsimply CONNECTED COVERING SPACES}

Let $M$ be an analytic Riemannian manifold such that every geodesic is contained in a compact (immersed) flat and let $\pi: \tilde{M} \rightarrow M$ be the universal covering. The aim of this section is to factorize $\pi$ through an intermediate but convenient covering.

By Proposition 2.1 we can write $\tilde{M}=\mathbb{R}^{k} \times \tilde{N}$, where $\tilde{N}=C_{1} \times \cdots \times C_{r} \times$ $S_{1} \times \cdots \times S_{l}$, where $C_{i}$ is a (simply connected) space all of whose geodesics are closed and $S_{j}$ is a (simply connected) irreducible symmetric space of the compact type and rank at least $2(i=1, \ldots, r, j=1, \ldots, l)$. Let $\pi_{C_{i}}$ and $\pi_{S_{j}}$ denote the projection of $\tilde{N}$ onto each one of its factors. One has, from the de Rham Splitting Theorem, that $I(\tilde{M})=I\left(\mathbb{R}^{k}\right) \times I(\tilde{N})$, where $I$ denotes the group of isometries. Let $G \subset I(\tilde{M})$ be the covering group associated with $\pi: \tilde{M} \rightarrow M$. Let $p r_{1}: G \rightarrow I\left(\mathbb{R}^{k}\right)$ be the projection and let

$$
G^{\prime}=\operatorname{ker}\left(p r_{1}\right)=\left\{g \in G: g=\left(i d_{\mathbb{R}^{k}}, \bar{g}\right), \bar{g} \in I(\tilde{N})\right\} .
$$

If $A$ is an isometry of $\mathbb{R}^{k}$ we will denote by $r(A)$ its projection to the orthogonal group $O(k)$ (i.e., $A=\tau \circ r(A)$ for some translation $\tau$ ). The Lie group morphism $r \circ p r_{1}: G \rightarrow O(k)$ induces a morphism $\bar{r}$ from the group $G / G^{\prime}$ into $O(k)$. 
Part (i) of the following lemma is standard and follows from the compactness of non-Euclidean factors. Parts (ii) and (iii) are a consequence of part (i) and the First Theorem of Bieberbach (see e.g. [C]).

Lemma 3.1. Under the general assumptions of this section. Then,

(i) $\operatorname{pr}_{1}(G)$ acts proper discontinuously on $\mathbb{R}^{k}$ (i.e. it is a crystallographic group).

(ii) $r\left(p r_{1}(G)\right)$ is finite.

(iii) The kernel of $\left.r\right|_{p r_{1}(G)}$ is a lattice of $\mathbb{R}^{k}$.

Let $\Gamma=\operatorname{ker}(\bar{r})$. Then $\left(G / G^{\prime}\right) / \Gamma$ is isomorphic to $\bar{r}\left(G / G^{\prime}\right)=r\left(p r_{1}(G)\right)$ and therefore finite.

Let us consider the cover $p: \tilde{M} \rightarrow M_{0}:=\tilde{M} / G^{\prime}$. Then, $G_{0}=G / G^{\prime}$ is naturally identified with a (discrete) subgroup of $I\left(M_{0}\right)$. By means of this identification $\Gamma$ turns out to be a normal subgroup of $G_{0}$. We have the following covers:

$$
\tilde{M} \stackrel{p}{\rightarrow} M_{0} \stackrel{\tilde{\pi}}{\rightarrow} M_{0} / \Gamma
$$

where $\tilde{\pi}$ is the projection to the quotient. Observe the following properties:

a) $M_{0}=\mathbb{R}^{k} \times N$, where $N$ is a compact space covered by $\tilde{N}$.

b) The group $\Gamma$ is given by

$$
\Gamma=\left\{(t, h) \in G: t \text { is a translation of } \mathbb{R}^{k} \text { and } g \in I(N)\right\} .
$$

In particular, $\operatorname{pr}_{1}(\Gamma)=p r_{1}(G) \cap \tau\left(\mathbb{R}^{k}\right)$ is a lattice of $\mathbb{R}^{k}$, where $\tau\left(\mathbb{R}^{k}\right)$ denotes the group of translations.

c) If $\left(t, h_{1}\right),\left(t, h_{2}\right) \in \Gamma$, then $h_{1}=h_{2}$. In other words, the group morphism $p r_{1}: \Gamma \rightarrow \tau\left(\mathbb{R}^{k}\right)$ is injective.

d) The manifold $M_{0} / \Gamma$ is a finite cover of $M$ and so every geodesic of $M_{0} / \Gamma$ is contained in a compact flat. In fact, regarding $G_{0} / \Gamma$ as a subgroup of $I\left(M_{0} / \Gamma\right)$, we have the following isometries:

$$
\frac{M_{0} / \Gamma}{G_{0} / \Gamma} \simeq M_{0} / G_{0} \simeq \frac{\tilde{M} / G^{\prime}}{G / G^{\prime}} \simeq \tilde{M} / G=M .
$$

Now let $z$ belong to the lattice $\mathcal{Z}:=p r_{1}(\Gamma) \subset \tau\left(\mathbb{R}^{k}\right)$ and let $\phi(z)$ be the unique $h \in I(N)$ such that $(z, h) \in \Gamma$. Then, $\phi: p r_{1}(\Gamma) \rightarrow p r_{2}(\Gamma)$ is an epimorphism. Thus, any element of $\Gamma$ can be written as $(t, \phi(t))$ where $\tau \in \mathcal{Z}$. Summarizing, we have the following result.

Lemma 3.2. Let $M$ be an analytic Riemannian manifold such that every geodesic is contained in a compact flat. Then, $M$ admits a finite cover $\bar{M}=\left(\mathbb{R}^{k} \times N\right) / \Gamma($ so, with every geodesic contained in a compact flat) which has the following properties:

(i) The universal cover of $N$ is a product of higher rank symmetric spaces of the compact type and (simply connected) analytic spaces all af whose geodesics are closed.

(ii) $\Gamma$ is a subgroup of $\tau\left(\mathbb{R}^{k}\right) \times I(N)$ acting in a proper and discontinuous way on $\mathbb{R}^{k} \times N$. Moreover, $\mathcal{Z}=\{t:(t, g) \in \Gamma$ for some $g \in I(N)\}$ is a lattice of $\mathbb{R}^{k}$ and there exists a (group) morphism $\phi: \mathcal{Z} \rightarrow I(N)$ such that any element of $\Gamma$ can be written as $(t, \phi(t))$ for some translation $t$. (In particular, $\Gamma$ is abelian.) 


\section{The DECOMPOSITION}

Let $M$ be an analytic (compact) manifold all of whose geodesics are contained in compact flats. From Lemma 3.2 we may assume, by replacing $M$ by a finite cover if necessary, that $M=\left(\mathbb{R}^{k} \times N\right) / \Gamma$, where $\Gamma=\{(t, \phi(t)): t \in \mathcal{Z}\}$ and $N$ are as in Lemma 3.2. Let $\left\{z_{1}, \cdots, z_{k}\right\}$ be a $\mathbb{Z}$-basis of $\mathcal{Z}$ (i.e., a basis over the integers). If, for every $i \in\{1, \cdots, k\}, \phi\left(\mathbb{Z} . z_{i}\right)$ is finite (or equivalently discrete, since $N$ is compact), then ker $\phi$ is also a lattice. Then, $\mathbb{R}^{k} / \operatorname{ker} \phi=T^{k}$ is a torus and $M$ is covered by $T^{k} \times N$ and so, by $T^{k} \times \tilde{N}$, as we wanted to show. So, we may assume, in order to prove Theorem $\mathrm{A}$, that $\phi\left(\mathbb{Z} z_{i_{0}}\right)$ is not finite, for some $i_{0} \in\{1, \cdots, g\}$. Without loss of generality we assume $i_{0}=1$. We shall derive, in this case, a contradiction. Let $K$ be the connected component of the identity of the closure, in $I(N)$, of $\phi\left(\mathbb{Z} z_{1}\right)$. Then, $\operatorname{dim}(K) \geq 1$, since $\phi\left(\mathbb{Z} z_{1}\right)$ is not discrete. Let $c$ be a geodesic in $\mathbb{R}^{k} \times N$ with $c(0)=(0, q)$ and let us consider the geodesic $\pi \circ c$ in $M$, where $\pi$ is the projection to the quotient space. We have, from the assumptions, that the compact set, which consists of closure of $\pi \circ c$, is the image of a flat $i: \mathbb{R}^{j} \rightarrow M$ (which factorizes through a torus). We may assume that $i(0)=\pi(c(0))$. The lift $\tilde{i}: \mathbb{R}^{j} \rightarrow \mathbb{R}^{k} \times N$ of $i$ with $\tilde{i}(0)=c(0)$ is also a flat.

Now choose the geodesic $c$ in $\mathbb{R}^{k} \times N$ to be of the form

$$
c(t)=\left(t z_{1}, p(\beta(t))\right),
$$

where $\beta(t)$ is a closed geodesic in $\tilde{N}$ of period (not necessarily the least one) 1 , where $p$ denotes the projection from $\tilde{N}$ into $N$. Then, for any $d \in \mathbb{Z}$, we have that

$$
\pi\left(\left(d z_{1}, p(\beta(d))\right)\right)=\pi\left(\left(d z_{1}, q\right)\right)=\pi\left(g^{d} \cdot\left(d z_{1}, q\right)\right)=\pi\left(\left(0, \phi\left(-d z_{1}\right) \cdot q\right)\right)
$$

where $g \in \Gamma$ is the isometry of $\mathbb{R}^{k} \times N$ which maps $(u, n) \mapsto\left(u-z_{1}, \phi\left(-z_{1}\right) \cdot n\right)$. Now let $W$ be a neighborhood of $(0, q)$ such that $\pi_{\left.\right|_{W}}$ is a diffeomorphism onto its image. It is now not difficult to see that

$$
\pi((0, K \cdot q) \cap W) \subset i\left(\mathbb{R}^{j}\right) \cap \pi(W) .
$$

Lemma 4.1. Let $U$ be an open subset of $S \tilde{N}$, the unit tangent bundle of $\tilde{N}$. Then there exists a closed geodesic $\beta$ in $\tilde{N}$ with period 1 such that, if $c(t)=\left(t z_{1}, p(\beta(t))\right)$, where $p$ is the projection of $\tilde{N}$ onto $N$, then

a) the flat $c l(\pi(c(\mathbb{R})))$ does not intersects itself at $\pi(c(0))$, and

b) $\beta^{\prime}(0) /\left\|\beta^{\prime}(0)\right\| \in U$.

In other words, the set $W$ of initial directions of closed geodesics $\beta$ with period 1 whose induced (as above) compact flat in $M$ has no self intersection in $\pi\left(c_{\beta}(0)\right)$ is dense in $S \tilde{N}$.

Proof. Each de Rham factor of $\tilde{N}$ is either a manifold of closed geodesics or a symmetric space of the compact type and rank $\geq 2$; thus the set consisting of those $v \in T \tilde{N}$ for which the geodesic $\gamma_{v}$ is closed is dense in $\tilde{N}$. By means of an appropriate reparametrization we can find a closed geodesic $\bar{\beta}$ of period 1 in $\tilde{N}$ such that $\bar{\beta}^{\prime}(0) /\left\|\bar{\beta}^{\prime}(0)\right\| \in U$. Let $\bar{c}(t)=\left(t z_{1}, p(\bar{\beta}(t))\right.$ and choose a point $s$, in the compact flat $c l(\pi(\bar{c}(\mathbb{R})))$, which is arbitrarily close to $\pi(\bar{c}(0))$ and such that this flat has no self intersection at this point. Now consider the parallel transport $v$ of $d \pi\left(\bar{c}^{\prime}(0)\right)$ along a short curve $u(t)$ in the flat from $\pi(\bar{c}(0))$ to $s$. One obtains that $\operatorname{cl}\left(\gamma_{v}(\mathbb{R})\right)=\operatorname{cl}(\pi(\bar{c}(\mathbb{R})))$. Now let $\tilde{u}(t)$ be the lift to $\mathbb{R}^{k} \times \tilde{N}$ of $u(t)$ with 
$\tilde{u}(0)=(0, \bar{\beta}(0))$ and let $\tilde{v}=\left(z_{1}, v_{1}\right)$ be the parallel transport of $\left(z_{1}, \bar{\beta}^{\prime}(0)\right)$ along $\tilde{u}$. Then, $d \pi\left(\left(0, d p\left(v_{1}\right)\right)\right)=v$ and the geodesic $\beta:=\gamma_{v_{1}}$ of $\tilde{N}$ is closed. If $s$ is sufficiently close to $\pi(c(0))$, then $v_{1} /\left\|v_{1}\right\|=\beta^{\prime}(0) /\left\|\beta^{\prime}(0)\right\| \in U$ and $c(t)=\left(t z_{1}, p(\beta(t))\right.$ satisfies (a) and (b) of the lemma. Note that $\beta$ has period 1 , since each of its projections to the de Rham factors has this property. In fact, for symmetric spaces one has to observe that the parallel transport can be achieved by the differential of an isometry. For spaces all of whose geodesics are closed this is a consequence of the fact that all geodesics have the same length (perhaps we have to replace $\beta$ by a geodesic whose initial condition is an integer multiple $\beta^{\prime}(0)$, so that the projection of this geodesic to a factor of closed geodesics has the common period).

Proof of Theorem A. We keep the notation and assumptions of this section. As was remarked at the beginning of this section it suffices to prove that $K$ is finite. Let us then assume that $\operatorname{dim} K \geq 1$. We will derive a contradiction. Now let $X \neq 0$ be in the Lie algebra of $K$, and let $\tilde{X}$ be the induced Killing field in the universal cover $\tilde{N}$ of $N$. Observe that in an open and dense subset of $\tilde{N}$ the field $\tilde{X}$ is nonzero. Let $W$ be the dense subset of $S \tilde{N}$ given by Lemma 4.1, let $C$ be a de Rham factor of $\tilde{N}$ which is of closed geodesics and let $\pi_{C}$ be the corresponding projection. Let $w \in W$ and $c(t)=\left(t . z_{1}, p(\beta(t))\right)$, where $\beta^{\prime}(0)=w$, and let $i: \mathbb{R}^{j} \mapsto \mathbb{R}^{k} \times \tilde{N}$ be the lift of the flat $c l(\pi(c(\mathbb{R})))$ with $i(0)=(0, \beta(0))$. This flat has no self intersection at $\left(0, \beta^{\prime}(0)\right)$, and $\left(z_{1}, \tilde{X}(\beta(0))\right),\left(z_{1}, \beta^{\prime}(0)\right)$ belong both to its tangent space at this point (see the paragraph just before Lemma 4.1). The projection $\pi_{C} \circ i\left(\mathbb{R}^{j}\right)$, being a flat in $C$, must be a closed geodesic (otherwise there would exist a nonclosed geodesic in $C)$. Then, the projections of $\tilde{X}(\beta(0))$ and $\beta^{\prime}(0)$ to the tangent space of $C$ must be linearly dependent. This shows that for every $w \in W, d \pi_{C}(w)$ and $d \pi_{C}(\tilde{X}(p r(w)))$ (where $p r$ is the projection to the base point) are linearly dependent. This is impossible, by the density of $W$, unless the $C$-component of $\tilde{X}$ vanishes. Now let $S$ be a (compact) symmetric factor of $\tilde{N}$ and let $\pi_{S}$ be the projection of $\tilde{N}$ onto $S$. As before, for every $w \in W$, the projections of $w$ and $\tilde{X}(\operatorname{pr}(w))$ to the tangent space of $S$ are tangent to the flat $\pi_{S} \circ i\left(\mathbb{R}^{j}\right)$. Thus, the sectional curvature $K_{S}\left(d \pi_{S}(w), d \pi_{S}(\tilde{X}(p r(w)))\right)=0$ for all $w \in W$ and consequently, by the density, for all $w \in T \tilde{N}$. So, the Ricci curvature $\operatorname{Ric}_{S}\left(\left(d \pi_{S}(\tilde{X}(q))\right)\right)=0$ for all $q \in \tilde{N}$. Since $S$ is an Einstein (non-Ricci flat) manifold, we obtain that the $S$-component of $\tilde{X}$ vanishes. Then, $\tilde{X}=0$ which is a contradiction.

Remark 4.2. Let $C$ be a space all of whose geodesics are closed (which must be all of the same length by $[\mathrm{BS}$ ). Let $\gamma$ be a geodesic in $C$ and let $J$ be a Jacobi field along $\gamma$ which is perpendicular to the geodesics and such that $J(0)=0$. Since $J$ can be obtained by variation of geodesics stating all at $\gamma(0)$ we obtain that $J(\tau)=0$, where $\tau$ is the common period of all geodesics. Then, there cannot exist a parallel Jacobi field $e$ along $\gamma$. Otherwise the field $t \mapsto t e(t)$ is Jacobi and vanishes only at 0 . Then, $\operatorname{rank}(C)=1$, where $\operatorname{rank}$ denotes the rank in terms of Jacobi fields.

Remark 4.3. Let $M$ be a space all of whose geodesics are contained in compact flats. The analyticity is never used after proving that the universal cover of $M$ splits, up to a Euclidean factor, as a product of spaces all of whose geodesics are closed by higher rank symmetric spaces. So, if the conjecture in [MO] is true, Theorem A is also true without assuming analyticity. 


\section{REFERENCES}

[B] W. Ballmann, Nonpositively curved manifolds of higher rank, Ann. of Math. (2) 122 (1985), 597-609. MR 87e:53059

[Be] M. Berger, Sur les groupes d'holonomie homogène des variétés à connexion affine et des variétés riemanniennes, Bull. Soc. Math. France 83 (1953), 279-330. MR 18:149a

[Bs] A. Besse, Manifolds all of whose geodesics are closed, Ergebnisse der Math. 93, SpringerVerlag, Berlin 1978. MR 80c:53044

[BS] K. Burns and R. Spatzier, Manifolds of nonpositive curvature and their buildings, Inst. Hautes Études Sci. Publ. Math. 65 (1987), 35-59. MR 88g:53050

[C] L. Charlap, Bieberbach groups and flat manifolds, Universitext, Springer-Verlag, New York, 1986. MR 88j:57042

[EH] P. Eberlein and J. Heber, A differential geometric characterization of symmetric spaces of higher rank, Inst. Hautes Études Sci. Publ. Math. 71 (1990), 33-44. MR 91j:53022

[EO] J. Eschenburg and C. Olmos, Rank and symmetry of Riemannian manifolds, Comment. Math. Helvetici 69 (1994), 483-499. MR 96d:53033

[HPTT] E. Heintze, R. Palais, C.-L.Terng and G. Thorbergsson, Hyperpolar actions and $k$-flats homogeneous spaces, J. Reine Angew. Math. 454 (1994), 163-179. MR 96b:53062

[MO] B. Molina and C. Olmos, Manifolds all of whose flats are closed, J. Differential Geometry 45 (1997), 575-592. MR 98d:53059

[S] J. Simons, On the transitivity of holonomy systems, Ann. of Math. 76 (1962), 213-234. MR 26:5520

[SS] R. Spatzier and M. Strake, Some examples of higher rank manifolds of nonnegative curvature, Comment. Math. Helvetici 65 (1990), 299-317. MR 91g:53044

Fa.M.A.F., Universidad Nacional de Córdoba, Ciudad Universitaria, 5000 Córdoba, ARgENTINA

E-mail address: molina@math.uni-augsburg.de

Fa.M.A.F., Universidad Nacional de Córdoba, Ciudad Universitaria, 5000 Córdoba, Argentina

E-mail address: olmos@mate.uncor.edu 